\title{
ADHS-Symptome und emotionales Wohlbefinden im Jugendalter und die Rolle ausgewählter Schutzfaktoren
}

\author{
Annette Krauss \\ Interkantonale Hochschule für Heilpädagogik $(\mathrm{HfH})$, Zürich
}

Zusammenfassung: Angelehnt an die entwicklungspsychologische Resilienzforschung wird im vorliegenden Artikel der Zusammenhang zwischen selbsteingeschätzten ADHS-Symptomen, emotionalem Wohlbefinden und ausgewählten Schutzfaktoren bei Schülern und Schülerinnen der Sekundarstufe II in der Deutschschweiz untersucht. 907 Jugendliche und junge Erwachsene zwischen 14 und 24 Jahren $(M=17.69 ; S D=1.66 ; 58,9 \%$ weiblich) wurden mittels Fragebogen im Klassenverband befragt. Multiple hierarchische Regressionsanalysen legen den Schluss nahe, dass ADHS-Symptome einen substanziellen negativen Effekt auf das emotionale Wohlbefinden haben und dass das eigene Kompetenzerleben, das Vorhandensein emotionaler Unterstützung durch nahe Bezugspersonen sowie durch Mitschülerinnen und Mitschüler den negativen Effekt der Symptome auf das Wohlbefinden teilweise zu kompensieren vermögen. Die vorliegende Untersuchung unterstreicht die Relevanz der Stärkung des Kompetenzerlebens und die Notwendigkeit, einen Blick auf die verfügbare emotionale Unterstützung bei Betroffenen zu werfen.

Schlüsselbegriffe: ADHS-Symptome, emotionales Wohlbefinden, Jugendalter, Schutzfaktoren, Resilienz

\section{ADHD symptoms and emotional well-being in adolescence and the role of selected protective factors}

Summary: Based on developmental psychological research on resilience, this article examines the relationship between self-rated ADHD symptoms, emotional well-being, and selected protective factors among secondary school students in German-speaking Switzerland. 907 adolescents and young adults between 14 and 24 years of age $(M=17.69 ; S D=1.66 ; 58.9 \%$ female) were surveyed by questionnaires in class. Multiple hierarchical regression analyses suggest that ADHD symptoms have a substantial negative effect on emotional well-being and that one's own sense of competence, emotional support from close caregivers, and from classmates can partially compensate for the negative effect of symptoms on well-being. The present study highlights the relevance of strengthening the sense of competence and the need to take a look at the available emotional support among affected individuals.

Keywords: ADHD symptoms, emotional well-being, adolescence, protective factors, resilience

Eine der am häufigsten vorkommenden psychischen Störungen bei Kindern und Jugendlichen im Schulalter ist die Aufmerksamkeitsdefizit-/ Hyperaktivitätsstörung (ADHS) mit den Kardinalsymptomen Unaufmerksamkeit, Hyperaktivität und Impulsivität (vgl. DSM-V; Falkai \& Wittchen, 2015). Die Kernsymptomatik ist dimensional in der Population verteilt (Bitto, Mörstedt, Faschina \& Stieglitz, 2017) und kann auch im subklinischen Bereich bedeutende Auswirkungen auf das tägliche Erleben und Verhalten haben (Whalen, Jamner, Henker, Delfino \& Lozano, 2002). Die Adoleszenz und der Übergang ins Erwachsenenalter können für be- 
troffene Jugendliche eine besondere Herausforderung darstellen. So ist bekannt, dass ADHSSymptome ein Risikofaktor für psychosoziale Problematiken und weitere psychische Störungen im Jugendalter darstellen (Meinzer et al., 2013; Schmidt, Brähler, Petermann \& Koglin, 2012; Tischler, Schmidt, Petermann \& Koglin, 2010). Dies macht effektive Behandlungs- und Präventionsstrategien für Betroffene unabdingbar.

Obwohl eine Fülle von Studien über die negativen Auswirkungen berichtet, die Jugendliche mit ADHS-Symptomen in verschiedenen Lebensbereichen erleben, ist das komplexe Störungsbild doch sehr heterogen, und eine Minderheit der Betroffenen scheint in verschiedenen Lebensbereichen erfolgreich und zufrieden zu sein (Lee, Lahey, Owens \& Hinshaw, 2008; Molina et al., 2009). Höchstwahrscheinlich tragen verschiedene Schutzfaktoren dazu bei, die negativen Auswirkungen der Symptomatik abzuschwächen. Das Konzept der Schutzfaktoren hat in den letzten Jahrzehnten zunehmend Verbreitung in Disziplinen wie der Klinischen Psychologie (Noeker \& Petermann, 2008), der Prävention und Gesundheitsförderung (Ravens-Sieberer, Wille, Bettge \& Erhart, 2007) sowie auch der (Sonder-) Pädagogik (Schwab \& Fingerle, 2013) gefunden und eignet sich auch, um Ansätze für eine multimodale Behandlungsund Präventionsstrategie für Jugendliche mit ADHS-Symptomen abzuleiten. Die frühere Forschung zu ADHS-Symptomen war lange Zeit primär defizitorientiert. Untersuchungen zu Schutzfaktoren bei Jugendlichen mit ADHS-Symptomatik gibt es noch vergleichsweise wenige.

Ziel der vorliegenden Untersuchung ist es, ausgehend von einem entwicklungspsychopathologischen Ansatz, den Einfluss des Risikofaktors ADHS-Symptomatik auf das emotionale Wohlbefinden bei Jugendlichen der Sekundarstufe II in der Schweiz zu untersuchen und insbesondere die Rolle ausgewählter Schutzfaktoren (Kompetenzerleben und emotionale Unterstützung) zu analysieren.

\section{ADHS-Symptome als Risikofaktor für das emotionale Wohlbefinden im Jugendalter}

Das Jugendalter stellt mit seinen Entwicklungsaufgaben (u. a. Ablösung vom Elternhaus, Übernahme von Autonomie und Selbstständigkeit, Ausbildung, Beziehungserfahrungen) und der verstärkten Abnahme von formalen Strukturen (Hurrelmann, 2007) Jugendliche mit ADHSSymptomen vor vielfältige Herausforderungen. Während die motorische Hyperaktivität in der Regel im Laufe der Jahre nachlässt, sind die Impulsivität und Aufmerksamkeitsstörungen im Jugendalter meist nach wie vor vorhanden (Kohn \& Esser, 2008). Damit zusammenhängende Probleme in der Selbstregulation, in organisatorischen Belangen und im Planen von längerfristigen Zielen (Martel, Nikolas \& Nigg, 2007) machen die Übernahme von Eigenverantwortlichkeit, die im Jugendalter vermehrt gefordert ist, schwierig.

Frustrationserfahrungen, die sich u. a. aufgrund von Schulleistungsproblemen (Frazier, Youngstrom, Glutting \& Watkins, 2007) sowie Problemen in der sozialen Interaktion (Edwards, Barkley, Laneri, Fletcher \& Metevia, 2001; Murray-Close et al., 2010) seit dem Kindesalter ergeben und über die Jahre kumulieren, können dazu führen, dass Jugendliche mit ADHS häufige negativ über ihre eigenen Fähigkeiten denken (Dvorsky, Langberg, Becker \& Evans, 2019; Krueger \& Kendall, 2001) und häufig dysfunktionale Stressbewältigungsstrategien anwenden (Brooks, 2011). Damit zusammenhängend ist oft auch das emotionale Wohlbefinden vermindert verglichen mit Gleichaltrigen ohne entsprechende Symptomatik (Baumgarten et al., 2019; Schmidt et al., 2012).

Trotz dieser vielfältigen Problematiken und dem hohen Leidensdruck der Betroffenen besteht in dieser Altersgruppe eine geringe Bereitschaft zur Fortsetzung der medikamentösen oder verhaltenstherapeutischen Behandlung (u. a. Bachmann, Philipsen \& Hoffmann, 2017). Daneben ist anzunehmen, dass es eine Reihe von Jugendlichen mit ADHS-Symptomen (im 
subklinischen Bereich) gibt, die von Auswirkungen der Symptomatik betroffen sind, aber keine Behandlung erhalten (Philipp-Wiegmann, Rösler, Retz, Schindelbeck \& Retz-Junginger, 2015). Vor diesem Hintergrund erscheint es wichtig, dass die Jugendlichen vom privaten wie auch schulischen Umfeld in ihrer Entwicklung gestärkt werden. Erkenntnisse zu relevanten Schutzfaktoren können hierbei leitend sein.

\section{ADHS-Symptome und relevante Schutzfaktoren}

Als Referenzrahmen für die vorliegende Untersuchung kann die entwicklungspsychopathologische Resilienzforschung herangezogen werden, die der Frage nachgeht, welche Einflussgrößen Kinder und Jugendliche in die Lage versetzen, sich trotz Belastungen und Risikofaktoren gesund zu entwickeln (Fergus \& Zimmerman, 2005; Masten, 2001). Aus einschlägigen Studien, wie beispielsweise der Kauai-Längsschnittstudie (Werner, 2004) oder der Mannheimer Risikokinderstudie (Laucht, 2012), konnten übereinstimmend relevante Schutzfaktoren bestimmt werden, die bei verschiedenen Formen von Widrigkeit puffernd wirken. Zu den zwei wichtigsten Schutzfaktoren für die psychische Gesundheit von Jugendlichen zählen ein positives Kompetenzerleben und die soziale Unterstützung von verschiedenen Seiten (Keyes, 2013; Masten, 2001; Ringdal, Espnes, Eilertsen, Bjørnsen \& Moksnes, 2020; Rueger, Malecki, Pyun, Aycock \& Coyle, 2016; Schwarzer \& Luszczynska, 2006; Vieno, Santinello, Pastore \& Perkins, 2007).

Mit einem positiven Kompetenzerleben ist der Glaube an die eigenen Fähigkeiten gemeint, mit Veränderungen, schwierigen Lebenssituationen und Gefühlen umgehen zu können (Sarubin et al., 2015). Das Konzept ist eng verwandt mit der Selbstwirksamkeit (Bandura, Freeman \& Lightsey, 1999) und zeigt auch konzeptuelle Überschneidungen mit anderen sozial-kognitiven Konstrukten wie beispielsweise den Kontrollüberzeugungen (locus of control; Rotter,
1966) oder dem Selbstwert. Querschnittsstudien sowie längsschnittliche Analysen geben Hinweise darauf, dass ein hohes (schulisches) Kompetenzerleben sowie ein hohes Kontrollerleben Jugendliche mit ADHS-Symptomen vor depressiven Symptomen (McQuade, Hoza, Waschbusch, Murray-Close \& Owens, 2011; Ostrander \& Herman, 2006; Schei et al., 2015 b) schützen können. Dvorsky et al. (2019) untersuchten den Verlauf des Selbstwerts von Jugendlichen mit ADHS während 18 Monaten und zeigten, dass Jugendliche mit vergleichsweise hohem und zunehmendem Selbstwert bis zum Alter von 15 Jahren u. a. weniger depressive Symptome aufwiesen. Die Autoren folgerten daraus, dass ein hohes Selbstwertgefühl eine positive Anpassung beim Vorliegen von ADHSSymptomen begünstigen kann.

Emotional unterstützende Beziehungen sind in der Adoleszenz eine wichtige Ressource für die Bewältigung der vielfältigen Anforderungen. Soziale Unterstützung hängt deshalb mit dem Wohlbefinden zusammen, weil sie zu positiven Emotionen und einem Gefühl des Selbstwerts und der Selbstwirksamkeit beiträgt und eine Pufferwirkung auf Stress ausüben kann (siehe auch Cohen \& Wills, 1985; Kienle, Knoll \& Renneberg, 2006). In einer klinischen Stichprobe von 149 Jugendlichen mit ADHS (Schei, Nøvik, Thomsen, Indredavik \& Jozefiak, 2015 a) ergaben sich Hinweise darauf, dass eine tragfähige soziale Unterstützung maßgeblich protektiv gegen emotionale Probleme wirken kann. Studien zeigen, dass insbesondere die emotionale Unterstützung durch nahestehende Personen (wie Eltern) sowie durch Gleichaltrige für das emotionale Wohlbefinden von Kindern und Jugendlichen mit ADHS-Symptomen zentral ist (Mastoras, Saklofske, Schwean \& Climie, 2018; McQuade et al., 2014; Powell et al., 2020). Bezüglich Lehrpersonen zeigen Studien mit Kindern mit ADHS sowie externalisierenden Auffälligkeiten, dass eine starke emotionale Beziehung zur Lehrperson u. a. mit weniger externalisierenden Verhaltensauffälligkeiten einige Jahre später zusammenhing (Hamre \& Pianta, 2001). 


\section{Fragestellung}

Bisher haben sich erst wenige Studien gezielt mit Schutzfaktoren im Zusammenhang mit ADHS-Symptomen im Jugendalter befasst. Die vorliegende Untersuchung soll einen Beitrag dazu leisten. Bei einer Stichprobe von Jugendlichen und jungen Erwachsenen auf Sekundarstufe II in der Schweiz wird anhand einer multiplen hierarchischen Regressionsanalyse untersucht, welche Auswirkungen ADHSSymptome auf das emotionale Wohlbefinden (als ein Indikator für eine erfolgreiche Anpassung im Jugendalter) haben und welche Rolle die Schutzfaktoren Kompetenzerleben sowie das Vorhandensein emotionaler Unterstützung von nahen Bezugspersonen, Mitschülern und Mitschülerinnen sowie Lehrpersonen dabei spielen.

\section{Methode}

\section{Projekt und Stichprobe}

Die vorliegende Untersuchung ist in das Projekt Enhanced Inclusive Learning (EIL; Schellenberg, Krauss, Pfiffner \& Georgi-Tscherry, 2020) eingebettet. Es verfolgte das Ziel, die Situation von Jugendlichen mit Beeinträchtigungen sowie sozio-emotionalen Auffälligkeiten in der Sekundarstufe II zu analysieren, wobei insbesondere die Thematik des Nachteilsausgleichs im Zentrum stand. Die Teilstudie zum Thema ADHS-Symptome untersuchte das Wohlbefinden und die Anforderungsbewältigung von betroffenen Jugendlichen. Dabei sollten auch Effekte von ausgewählten Schutzfaktoren auf eine erfolgreiche Anpassung bei ADHS-Symptomen untersucht werden. Im Rahmen des Projekts wurden im Zeitraum vom September 2018 bis Januar 2019 Jugendliche und junge Erwachsene aus Berufs- und Mittelschulen der Deutschschweiz mittels Fragebogen im Klassenverband befragt. Berufs- und Mittelschulen sind in der Schweiz auf der Sekundarstufe II angesiedelt. Insgesamt nahmen 66 Klassen (22 Mittelschulen und 44 Berufsschulen) aus elf Deutschschweizer Kantonen teil. Alle befragten Personen wurden mündlich sowie schriftlich über die Ziele und Inhalte der Befragung hingewiesen, und es wurde eine schriftliche Einwilligungserklärung zur Datenverwendung von allen Befragten eingeholt.
Die finale Stichprobe bestand aus 907 Jugendlichen und jungen Erwachsenen zwischen 14 und 24 Jahren ( $M=17.69 ; S D=1.66 ; 58,9 \%$ weiblich). Da der Fokus des Projekts auf dem Jugend- und frühen Erwachsenenalter lag, wurden Personen mit einem Alter über 24 Jahren nach der Datenerhebung aus der Stichprobe ausgeschlossen. Die Mittelschüler und -schülerinnen $(n=344)$ stammten aus dem 9. bis 13. Schuljahr und strebten eine gymnasiale Maturität ${ }^{1}$ an. Die Berufsschüler und -schülerinnen $(n=563)$ befanden sich in unterschiedlichen Ausbildungsjahren einer 3- bis 4-jährigen Ausbildung zum eidgenössischen Fähigkeitszeugnis $(\mathrm{EFZ})^{2}$ oder einer 2-jährigen Ausbildung zu einem eidgenössischem Berufsattest (EBA) ${ }^{3}$.

\section{Eingesetzte Instrumente}

Im Rahmen der schriftlichen Befragung wurden soziodemografische Angaben zum Geschlecht, Alter sowie Bildungsstand der Eltern (höchste absolvierte Ausbildung, 5-stufig) erhoben. In Anlehnung an Lampert, Hoebel, Kuntz, Müters und Kroll (2018) wurde bezüglich des Bildungsstands der Eltern der jeweils höhere berücksichtigt. Bei Jugendlichen, die nur für einen Elternteil Angaben zum Bildungsstand gemacht haben $(n=76)$, wurde diese Angabe herangezogen.

Um die ADHS-Symptomatik zu erfassen, wurde der Selbstbeurteilungsbogen ADHS des Diagnostik-Systems fir psychische Störungen nach ICD-10 und DSM-5 fuir Kinder-und Jugendliche (DISYPS-III; Döpfner \& Görtz-Dorten, 2017) eingesetzt. Die Skala besteht aus 20 Items zu den Bereichen Unaufmerksamkeit (Item 1-9), Hyperaktivität (Item 10-16) und Impulsivität (Item 17-20). Die Items wurden dabei auf einer vierstufigen Antwortskala (von 1=, ,gar nicht zutreffend“ bis $4=$ „, besonders zutreffend“) beurteilt. Die interne Konsistenz der Skala fiel gut aus $(\alpha=.88)$.

Das emotionale Wohlbefinden wurde mit der entsprechenden Subskala des KINDL-R-Fragebogen zur gesundheitsbezogenen Lebensqualität (Ravens-Sieberer $\&$ Bullinger, 2003) erhoben. Die Items sind 5-stufig Likert-skaliert mit den Antwortmöglichkeiten $1=$ =,nie“ bis $5=$,immer". Die interne Konsistenz der Skala liegt im unteren Bereich $(\alpha=.63)$, wobei in der Studie zur Gesundheit von Kindern und Jugendlichen in Deutschland (KiGGS; Bullinger, Brutt, Erhart, Ravens-Sieberer \& Bella Study Group, 2008) ein vergleichbarer Wert erreicht wurde. Da das Instrument als etabliertes Verfahren gilt, wurde es unverändert in die vorliegenden Analysen einbezogen. 
Das Kompetenzerleben wurde anhand der deutschsprachigen 10-Item Version der Connor-Davidson Resilience Scale (CD-RISC; Sarubin et al., 2015) erfasst. Die Skala misst den Glauben an die eigenen Fähigkeiten, mit Veränderungen, schwierigen Lebenssituationen und Gefühlen umgehen zu können. Zehn Aussagen werden hinsichtlich ihres Zutreffens innerhalb der letzten vier Wochen auf einer 5-stufigen LikertSkala beurteilt (von $0=$ „überhaupt nicht wahr“ bis $4=$ „fast immer wahr“). Die interne Konsistenz fiel gut aus $(\alpha=.82)$.

Das generelle Vorhandensein emotionaler Unterstützung durch Bezugspersonen wurde durch die Subskala „emotionale Unterstützung“ der Berliner Social Support Scale (BSSS; Schulz \& Schwarzer, 2003) erhoben. Vier Items werden auf einer Skala von $1=$ „stimmt nicht“ bis 4 = „stimmt genau“ erhoben. Die interne Konsistenz fiel gut aus $(\alpha=.87)$.

Die wahrgenommene emotionale Unterstützung durch Lehrpersonen sowie Mitschüler und Mitschülerinnen wurde durch zwei Skalen von Currie et al. (2014) in Anlehnung an Torsheim, Wold und Samdal (2016) erfasst. Drei bzw. vier Items werden auf einer Skala von $1=$,stimmt überhaupt nicht“ bis $5=$ =,stimmt genau" beantwortet. Die internen Konsistenzen fielen gut aus $(\alpha=.80$ bzw. .79).

\section{Statistische Auswertung}

Die statistischen Auswertungen erfolgten mithilfe des Statistikprogramms IBM SPSS Statistics 26. U. a. zur Identifizierung potenzieller Kovariaten (Geschlecht, Alter, höchster Ausbildungsstand der Eltern, Ausbildungstyp), die in die Regressionsmodelle einbezogen werden sollten, wurden als Erstes bivariate Korrelationen berechnet. Anschließend wurden zwei separate hierarchische Regressionsanalysen ${ }^{4}$ (blockweise) gerechnet, um den Einfluss der Schutzfaktoren auf das emotionale Wohlbefinden unter dem Einbezug von ADHS-Symptomen zu untersuchen. $\mathrm{Da}$ in der Literatur zu Resilienz zwischen kompensatorischen und protektiven Schutzfaktoren unterschieden wird (Dvorsky \& Langberg, 2016; Fergus \& Zimmerman, 2005; Masten, 2001), wurden nebst den unabhängigen Variablen auch Interaktionsterme in die Regressionsanalysen einbezogen ${ }^{5}$. Analog zur Resilienzliteratur (Fergus \& Zimmerman, 2005) wurden in einem ersten Schritt die Kontrollvariablen (demografische Variablen) eingeschlossen, im zweiten Schritt der Risikofaktor (ADHS-Symptome), im dritten Schritt die potenziellen Schutz- faktoren (Kompetenzerleben in Analyse I und emotionale Unterstützung von verschiedenen Seiten in Analyse II) und im vierten Schritt jeweils die Interaktionsterme von Risikofaktor und Schutzfaktoren. Dieses Vorgehen entspricht einer Moderationsanalyse.

Die unabhängigen Variablen und Kontrollvariablen wurden mittelwertzentriert und die Regressionen wurden auf Multikollinearität überprüft. Den Empfehlungen von Field (2018) folgend (durchschnittlicher Varianzinflationsfaktor VIF nahe bei 1 , kein Einzelwert über 10), gab es keine wesentlichen Probleme bezüglich Multikollinearität (alle VIF zwischen $1.01-1.18$ bei Analyse I und zwischen 1.05 und 1.27 bei Analyse II). Um signifikante Interaktionseffekte grafisch darzustellen, wurden simple slopes dargestellt und es wurden simple slope tests berechnet anhand von Werten, die jeweils eine Standardabweichung über und unter dem Mittelwert des Schutzfaktors liegen (Dawson, 2014).

Bei 46 Personen $(5,05 \%)$ konnten eine oder mehrere der berücksichtigten Subskalen wegen fehlender Werte nicht berechnet werden. Für die Regressionsanalysen wurde ein listenweiser Fallausschluss gewählt. Die einbezogene Stichprobe variierte deshalb zwischen 862 und 886 Personen.

\section{Ergebnisse}

Tabelle 1 zeigt die Mittelwerte und Standardabweichungen der untersuchten Variablen bzw. Skalen sowie die bivariaten Korrelationen. Von den Kontrollvariablen korrelierte das Geschlecht $(r=.10, p<.01)$ sowie das Alter $(r=-.08, p<.05)$ mit dem emotionalen Wohlbefinden. Die höchste Ausbildung der Eltern (5-stufig) sowie der Ausbildungstyp hingen hingegen nicht mit dem emotionalen Wohlbefinden zusammen und wurden dementsprechend nicht in die nachfolgenden Regressionsanalysen einbezogen. Die ADHS-Symptome korrelierten positiv mit dem Geschlecht $(r=.10$, $p<.01)$ und Alter $(r=.08, p<.05)$ und negativ mit den untersuchten Schutzfaktoren (von $r=-.11$ bis $r=-.29, p<.001)$ sowie dem emotionalen Wohlbefinden $(r=-.35, p<.001)$. Die untersuchten Schutzfaktoren hingen positiv mit dem emotionalen Wohlbefinden zusammen (von $r=.17$ bis $0.40, p<.001$ ). 
Tab. 1: Deskriptive Statistiken sowie bivariate Korrelationen

\begin{tabular}{|c|c|c|c|c|c|c|c|c|c|c|c|}
\hline & M & $S D$ & 2 & 3 & 4 & 5 & 6 & 7 & 8 & 9 & 10 \\
\hline 1: Geschlecht & 0.41 & 0.49 & .04 & .07 & -.01 & $.10^{* *}$ & $.19^{\star * \star}$ & $-.18^{* * *}$ & $-.08^{* *}$ & $.09^{* *}$ & $.10^{\star *}$ \\
\hline 2: Alter & 17.69 & 1.66 & & $-.10^{\star *}$ & .26 & $.08^{*}$ & $-.08^{*}$ & $-.07^{*}$ & $-.17^{\star \star *}$ & $.08^{*}$ & $-.08^{*}$ \\
\hline 3: höchste AB Eltern & 3.62 & 1.31 & & & -.26 & .03 & $.08^{*}$ & .04 & .06 & $-.14^{* * *}$ & -.02 \\
\hline 4: Ausbildungstyp & 0.62 & 0.49 & & & & -.03 & -.01 & -.06 & $-.12^{\star \star \star}$ & $.27^{\star \star \star}$ & -.02 \\
\hline 5: ADHS-Symptome & 0.67 & 0.42 & & & & & $-.29^{\star * *}$ & $-.24^{* * *}$ & $-.11^{* * *}$ & $-.13^{* * *}$ & $-.35^{* * *}$ \\
\hline 6: Kompetenzerleben & 2.79 & 0.54 & & & & & & $.16^{* * *}$ & $.11^{\star *}$ & $.13^{* * *}$ & $40^{* * *}$ \\
\hline 7: Unterstützung BP & 3.59 & 0.54 & & & & & & & $.25^{\star \star *}$ & $.11^{* * *}$ & $.37^{* * *}$ \\
\hline 8: Unterstützung MS & 3.99 & 0.63 & & & & & & & & $.24^{* * *}$ & $.22^{\star \star *}$ \\
\hline 9: Unterstützung LP & 3.83 & 0.69 & & & & & & & & & $.17^{\star \star *}$ \\
\hline 10: Emotionales WB & 3.90 & 0.69 & & & & & & & & & \\
\hline
\end{tabular}

Anmerkungen: $\mathrm{AB}=$ Ausbildung; $\mathrm{BP}=$ Bezugspersonen; $\mathrm{MS}=$ Mitschülerinnen und Mitschüler; $\mathrm{LP}=$ Lehrpersonen; $\mathrm{WB}=$ Wohlbefinden; Geschlecht: 0 = weiblich, 1 = männlich; Ausbildungstyp: $0=$ Mittelschule, 1 = Berufsschule.

${ }^{*} p<.05 ;{ }^{* *} p<.01 ;{ }^{* * *} p<.001$

Um mehr darüber zu erfahren, welche Effekte die ADHS-Symptome als Risikofaktor sowie die untersuchten Schutzfaktoren auf das emotionale Wohlbefinden haben, wurden zwei hierarchische multiple Regressionsanalysen gerechnet (Tabelle 2 für den Schutzfaktor Kompetenzerleben und Tabelle 3 für die Schutzfaktoren bzgl. emotionaler Unterstützung). In einem ersten Schritt wurden die soziodemografischen Faktoren Geschlecht und Alter in die Analysen eingeführt. Das Geschlecht erwies sich dabei als signifikanter Prädiktor für das emotionale Wohlbefinden $(\beta=.08, p<.01$ in Analyse 1 und $\beta=.19, p<.001$ in Analyse II). Konkret zeigte sich, dass das männliche Geschlecht mit einem höheren emotionalen Wohlbefinden einhergeht. In einem zweiten Schritt wurde der Risikofaktor ADHS-Symptome eingeführt, der sich ebenfalls als signifikanter Prädiktor erwies $(\beta=-.26$, $p<.001$ in Analyse 1 und $\beta=-.28, p<.001$ in Analyse II). In einem dritten und vierten Schritt wurden die ausgewählten Schutzfaktoren sowie die Interaktionsterme von den ADHS-Symptomen und Schutzfaktoren in die Analyse eingeführt.

In der Regressionsanalyse I konnte ein signifikanter Haupteffekt des Schutzfaktors Kompetenzerleben auf das emotionale Wohlbefinden $(\beta=.29, p<.001)$ sowie ein signifikanter, wenn auch schwacher Interaktionseffekt zwischen den ADHS-Symptomen und dem Kompetenzerleben $(\beta=.08, p<.01)$ gefunden werden. Dies legt den Schluss nahe, dass ein hohes Kompetenzerleben gegen die negativen Auswirkungen der ADHS-Symptome auf das emotionale Wohlbefinden kompensatorisch sowie auch protektiv wirken kann. Die simple slope tests zeigen, dass der negative Zusammenhang zwischen ADHS-

Tab. 2: Resultate der multiplen Regressionsanalyse I

\begin{tabular}{|l|l|l|l|l|}
\hline & & B & SE & \multicolumn{1}{|c|}{$\boldsymbol{\beta}$} \\
\hline Schritt 1: Sozio- & Geschlecht & .11 & .04 & $.08^{* *}$ \\
\hline demografische Faktoren & Alter & -.01 & .01 & -.03 \\
\hline Schritt 2: Risikofaktor & ADHS-Symptome & -.43 & .05 & $-.26^{* * *}$ \\
\hline Schritt 3: Schutzfaktoren & Kompetenzerleben & .37 & .04 & $.29^{* * *}$ \\
\hline Schritt 4: Interaktionen & Kompetenzerleben×ADHS & .22 & .08 & $.08^{* *}$ \\
\hline $\boldsymbol{R}^{2}$ adj. & & & & .23 \\
\hline
\end{tabular}

Anmerkungen: $N=886 .{ }^{* *} p<.01 ;{ }^{* * *} p<.001 ; \Delta R^{2}=.02$ für Schritt $1(p<.001) ; \Delta R^{2}=.13$ für Schritt $2(p<.001)$; $\Delta R^{2}=.08$ für Schritt $3(p<.001) ; \Delta R^{2}=.01$ für Schritt $4(p<.01)$. Geschlecht: $1=$ weiblich; $2=$ männlich. Konventionen zur Beurteilung der Veränderung in $R^{2}: .01=$ klein, $.06=$ mittel, $.14=$ stark (Cohen, Cohen, West \& Aiken, 2014). 
Tab. 3: Resultate der multiplen Regressionsanalyse II

\begin{tabular}{|c|c|c|c|c|}
\hline & $B$ & $S E$ & $\beta$ \\
\hline $\begin{array}{l}\text { Schritt 1: Sozio- } \\
\text { demografische Faktoren }\end{array}$ & $\begin{array}{l}\text { Geschlecht } \\
\text { Alter }\end{array}$ & $\begin{array}{r}.26 \\
-.01\end{array}$ & $\begin{array}{l}.04 \\
.01\end{array}$ & $\begin{array}{l}.19^{* * *} \\
-.02\end{array}$ \\
\hline Schritt 2: Risikofaktor & ADHS-Symptome & -.45 & .05 & $-.28 * * *$ \\
\hline Schritt 3: Schutzfaktoren & $\begin{array}{l}\text { Unterstützung BP } \\
\text { Unterstützung MS } \\
\text { Unterstützung LP }\end{array}$ & $\begin{array}{l}.37 \\
.13 \\
.04\end{array}$ & $\begin{array}{l}.04 \\
.03 \\
.03\end{array}$ & $\begin{array}{l}.29^{* * *} \\
.12^{* * *} \\
.04\end{array}$ \\
\hline Schritt 4: Interaktionen & $\begin{array}{l}\text { Unterstützung } \mathrm{BP} \times \mathrm{ADHS} \\
\text { Unterstützung } \mathrm{MS} \times \mathrm{ADHS} \\
\text { Unterstützung } \mathrm{LP} \times \mathrm{ADHS}\end{array}$ & $\begin{array}{r}.00 \\
-.06 \\
.00\end{array}$ & $\begin{array}{l}.08 \\
.08 \\
.06\end{array}$ & $\begin{array}{r}.00 \\
-.03 \\
.00\end{array}$ \\
\hline \multicolumn{4}{|l|}{$R^{2}$ adj. } & .24 \\
\hline
\end{tabular}

Anmerkungen: $N=862 .{ }^{* * *} p<.001 . \Delta R^{2}=.01$ für Schritt $1(p<.01) ; \Delta R^{2}=.13$ für Schritt $2(p<.001) ; \Delta R^{2}=.11$ für Schritt 3 ( $p<.001) ; \Delta R^{2}=.00$ für Schritt 4 ( $\left.p>.05\right)$. BP = Bezugspersonen; MS = Mitschülerinnen und Mitschüler; $\mathrm{LP}=$ Lehrpersonen. Geschlecht: 1 = weiblich; 2 = männlich. Konventionen zur Beurteilung der Veränderung in $R^{2}: .01=$ klein, $.06=$ mittel, $.14=$ stark (Cohen et al., 2014).

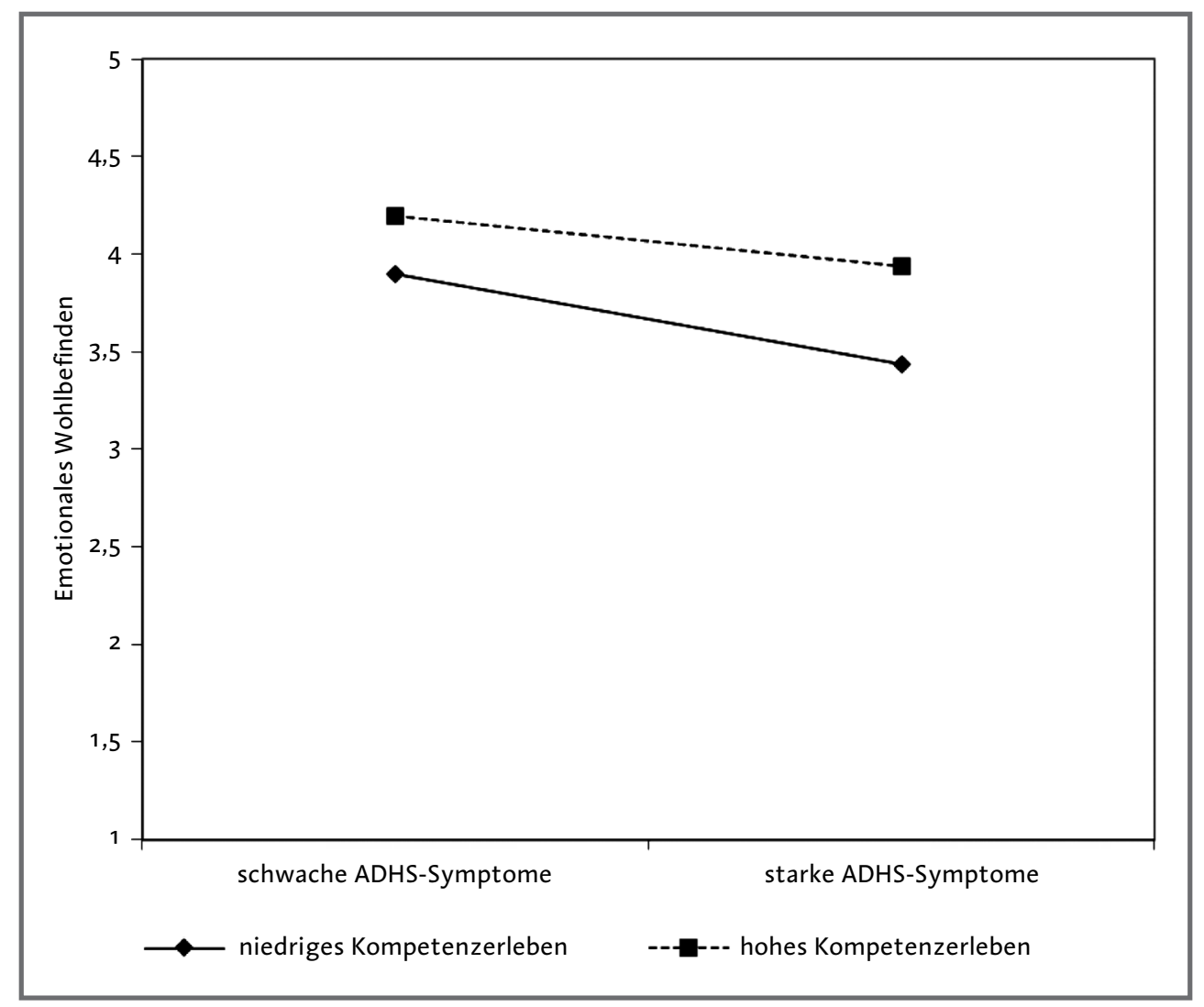

Abb. 1: Interaktionseffekt zwischen ADHS-Symptomen und Kompetenzerleben.

Anmerkungen: Bei einem hohen Kompetenzerleben fällt der Zusammenhang zwischen ADHS-Symptomen und emotionalem Wohlbefinden schwächer aus als bei einem niedrigen Kompetenzerleben. 
Symptomen und emotionalem Wohlbefinden bei einem hohen Kompetenzerleben schwächer ausfällt $(\beta=-.31 ; p<.001)$ als bei einem niedrigen Kompetenzerleben $(\beta=-.54 ; p<.001$; Abbildung 1). Durch die Prädiktoren konnte in der Regressionsanalyse I gesamthaft ein Varianzanteil von $R^{2}=.23$ aufgeklärt werden $(F(5,881)=52.99$, $p<.001)$.

In der Regressionsanalyse II konnten signifikante Haupteffekte des Vorhandenseins von emotionaler Unterstützung durch Bezugspersonen $(\beta=.29, p<.001)$ sowie durch Mitschülerinnen und Mitschüler $(\beta=.12, p<.001)$ auf das emotionale Wohlbefinden gefunden werden. Der stärkste Effekt konnte dabei im generellen Vorhandensein der emotionalen Unterstützung durch Bezugspersonen verortet werden. Der Effekt der Mitschüler und Mitschülerinnen fiel zwar kleiner aus, stellt aber trotzdem einen hochsignifikanten Einflussfaktor auf das emotionale Wohlbefinden im Modell dar. Die emotionale Unterstützung der Lehrpersonen erwies sich hingegen als nicht signifikant. Ebenso ergaben sich keine signifikanten Interaktionseffekte. Durch die Prädiktoren konnte gesamthaft ein Varianzanteil von $R^{2}=.24$ aufgeklärtwerden $(F(9,853)=31.96$, $p<.001)$.

\section{Diskussion}

Die Ergebnisse zeigen in Übereinstimmung mit anderen Studien (Baumgarten et al., 2019; Schmidt et al., 2012), dass ADHS-Symptome einen substanziellen negativen Effekt auf das emotionale Wohlbefinden von Jugendlichen und jungen Erwachsenen haben. Dieses Ergebnis überrascht nicht angesichts der spezifischen Schwierigkeiten in der Selbstregulation, des häufigen Auftretens von Komorbiditäten sowie der vielfältigen Herausforderungen im Alltag, die Betroffene erleben (Schmidt et al., 2012; Tischler et al., 2010). Es kann angenommen werden, dass sich die emotionalen Probleme bis ins Jugendalter verstärken. So zeigen Studien mit Kindern vergleichsweise weniger starke Zusammenhänge zwischen ADHS-Symptomen und emotionalem Befinden (u. a. Mastoras et al., 2018).
Die Ergebnisse zeigen weiter, dass das eigene Kompetenzerleben, das Vorhandensein der emotionalen Unterstützung durch eine nahestehende Bezugsperson und Mitschüler und Mitschülerinnen einen bedeutenden Effekt auf das emotionale Wohlbefinden im Regressionsmodell haben. Dies legt den Schluss nahe, dass diese Faktoren kompensatorisch gegen die negativen Auswirkungen der ADHS-Symptome auf das emotionale Wohlbefinden wirken können. Ein hohes eigenes Kompetenzerleben scheint zusätzlich auch protektiv zu wirken (ausgedrückt durch einen signifikanten Interaktionseffekt in der Regression).

Die negativen Auswirkungen der ADHSSymptome auf das emotionale Wohlbefinden können somit durch ein hohes wahrgenommenes Kompetenzerleben potenziell kompensiert und abgeschwächt werden. Dieses Ergebnis steht im Einklang mit Ergebnissen aus anderen Untersuchungen (Dvorsky et al., 2019; McQuade et al., 2011; Schei et al., 2015 b), die Aspekte einer positiven Selbstwahrnehmung als Schutzfaktor für das Wohlbefinden von Jugendlichen mit ADHS hervorheben. Aus diesen Erkenntnissen kann gefolgert werden, dass die Stärkung des Kompetenzerlebens bei von ADHS-Symptomen betroffenen Jugendlichen für deren emotionales Wohlbefinden zentral ist. Gerade in der Adoleszenz, in der stabilere Annahmen über die eigene Person getroffen werden (Cole, Jacquez \& Maschman, 2001), sollten Interventionen nebst der Verbesserung von spezifischen Bewältigungsfähigkeiten auch die Selbstwahrnehmung und die Überzeugungen bezüglich der eigenen Person in den Blick nehmen (Dvorsky et al., 2019; Major, Martinussen \& Wiener, 2013). Ziel dabei sollte nicht sein, dass die Herausforderungen und Schwierigkeiten von Betroffenen ausgeblendet werden und auch nicht, dass Betroffenen sich in ihren Kompetenzen überschätzen (in der Literatur unter dem Begriff, positive illusory bias diskutiert und längerfristig nicht adaptiv; Jia, Jiang \& Mikami, 2016). Vielmehr sollten 
sie wahrnehmen, dass auch sie ganz spezifische Stärken und Bewältigungsfähigkeiten haben. Damit könnte auch erreicht werden, dass sie weniger dysfunktionale Stressbewältigungsstrategien (wie beispielsweise sozialen Rückzug) anwenden, was häufig mit einem negativen Kompetenzerleben gekoppelt ist (Brooks, 2011).

Bezüglich emotionaler Unterstützung konnte der stärkste potenziell kompensatorisch wirkende Effekt beim Vorhandensein der emotionalen Unterstützung von nahen Bezugspersonen verortet werden. Die emotionale Unterstützung von Mitschülern und Mitschülerinnen zeigt ebenso einen signifikanten Effekt auf das emotionale Wohlbefinden im Regressionsmodell. Beide Effekte gelten unabhängig von den ADHS-Symptomen für alle Jugendlichen und fallen in Übereinstimmung mit Untersuchungen aus, die die Wichtigkeit des Vorhandenseins der emotionalen Unterstützung durch nahe Bezugsperson und Gleichaltrigen für die psychische Gesundheit von Jugendlichen (z. B. Ringdal et al., 2020) und spezifisch für Kinder und Jugendliche mit ADHS-Symptomen (Mastoras et al., 2018; Powell et al., 2020) zeigen. Die emotionale Unterstützung vonseiten der Lehrpersonen zeigt zwar bivariate Zusammenhänge zum emotionalen Wohlbefinden $(r=.17$, $p<.001)$, in der multiplen Regression unter Hinzunahme der anderen Prädiktoren ergibt sich hingegen kein signifikanter Effekt auf das emotionale Wohlbefinden mehr. Wenn man bedenkt, dass die Jugendlichen in der Sekundarstufe II mit einer Vielzahl von Lehrpersonen interagieren, erscheint es nachvollziehbar, dass der Effekt der emotionalen Unterstützung von Lehrpersonen im Vergleich zum Kindesalter (z. B. Ritter, Bilz \& Melzer, 2016) eher abnimmt. Zudem geben Untersuchungen (Demaray, Malecki, Davidson, Hodgson \& Rebus, 2005) Hinweise darauf, dass die wahrgenommene Unterstützung durch die Lehrpersonen in einem stärkeren Zusammenhang mit schulbezogenen Variablen, wie beispielsweise schulischen Leistungen, steht als mit emotionalen Merkmalen.
Gerade weil Jugendliche mit ADHS-Symptomen oftmals Schwierigkeiten in den Beziehungen $\mathrm{zu}$ Erwachsenen und Gleichaltrigen aufweisen (Murray-Close et al., 2010), sollte bei Betroffenen ein besonderer Bick darauf geworfen werden, welche emotionale Unterstützung für sie verfügbar ist. Dass keine protektiven Effekte (i.S.v. Interaktionseffekten) von emotionaler Unterstützung in den Analysen nachgewiesen werden konnten, schmälert die gefundenen Haupteffekte der emotionalen Unterstützung von Nahestehenden sowie Gleichaltrigen nicht. So wurde auch von Masten (2001) festgehalten, dass kompensatorische Faktoren (i. S. v. Haupteffekten) wichtige Einflussfaktoren darstellen, da sie kumulativ gegen negative Auswirkungen von Risikofaktoren wirken können. Bei Betroffenen von ADHS-Symptomen, die verschiedensten Herausforderungen gegenüberstehen, sind jegliche kompensierende Faktoren relevant, auf die im Rahmen von Behandlungs- und Präventionsstrategien fokussiert werden kann.

Im Zuge der schulischen Inklusion steigt der Anteil von Schülerinnen und Schülern mit Verhaltensauffälligkeiten an allgemeinen Schulen zusätzlich an. Dies macht es notwendig, die beiden Handlungsfelder Bildung und Gesundheit zusammen zu denken und effektive Strategien zum Umgang mit den vorliegenden Verhaltensauffälligkeiten zu entwickeln und anzuwenden. Verschiedene Autoren (u. a. Castello, 2017; Fabiano \& Pyle, 2019; Hanisch et al., 2019) schlagen für die pädagogische Praxis an Schulen bei ADHS-Symptomen eine gestufte Vorgehensweise mit evidenzbasierten Maßnahmen je nach Symptomstärke und Belastungserleben vor. Nebst universellen präventiven Maßnahmen (wie beispielsweise der Förderung sozial-emotionaler Kompetenzen, die u. a. zu einem positiven Selbstkonzept beitragen sollen; Casale, Hennemann \& Hövel, 2014) werden auf den nachfolgenden Stufen bei Jugendlichen mit stärkeren Auffälligkeiten eine intensivere selektive und indizierte Förderung durchgeführt. Da die emotionale Unterstützung, insbesondere auch von Mitschülerinnen und Mitschülern, wesentlich zum emotionalen Wohlbefinden 
von Jugendlichen mit ADHS-Symptomen beiträgt, können auch Peer-Ansätze (z. B. Peer-Mentoring) als vielversprechend angesehen werden. In verschiedenen Studien (u. a. Haft, Chen, LeBlanc, Tencza \& Hoeft, 2019) haben sich solche Ansätze bereits als vielversprechende Interventionen für die Verbesserung des emotionalen Wohlbefindens wie auch des Kompetenzerlebens bei Jugendlichen mit ADHS-Symptomen erwiesen. Empirische Wirksamkeitsbelege bezüglich Schulinterventionen sind in Hinblick auf das Jugendalter im Vergleich zum Kindesalter jedoch generell noch rar und sollten in Zukunft vermehrt in den Blick genommen werden.

Bei Jugendlichen mit starken ADHSSymptomen ist eine multimodale Behandlung angezeigt, die therapeutische Settings und Psychoedukations-, Selbstmanagements- und Coachingelemente beinhaltet (vgl. Linderkamp, Hennig \& Schramm, 2011; Rademacher, Walter \& Döpfner, 2002). Dabei sollten Lehrpersonen und Eltern aktiv in den Prozess miteinbezogen werden, da (verhaltens-)therapeutische Maßnahmen ein Übungsfeld zu Hause oder in der Schule bedingen, in dem neu erlernte Verhaltensweisen getestet werden können.

\section{Limitationen}

Obwohl es sich bei der vorliegenden Stichprobe um eine selektive handelt (es wurden gezielt Klassen ausgewählt, in denen jeweils mindestens ein Jugendlicher bzw. eine Jugendliche mit Nachteilsausgleich vertreten war), kann doch davon ausgegangen werden, dass es sich näherungsweise um eine repräsentative Stichprobe von Schulklassen auf Sekundarstufe II handelt, was das Auftreten von ADHS-Symptomen betrifft. So befand sich unter den Mittelschulbzw. Berufsschulklassen jeweils durchschnittlich nur eine Person, die einen Nachteilsausgleich erhielt, im Großteil der Fälle (61,7\% der Nachteilsausgleichfälle) für eine Lese-Rechtschreibstörung. In die vorliegenden Analysen wurden alle Schülerinnen und Schüler der Klasse einbezogen, also auch solche mit Nachteilsausgleich. Die Ergebnisse der Analysen verändern sich nur unwesentlich, wenn die Jugendlichen mit Nachteilsausgleich aus den Analysen ausgeschlossen werden.

Es ist weiter anzumerken, dass die Einschätzungen bezüglich ADHS-Symptomen ausschließlich auf Selbstauskünften der Jugendlichen beruhen, ohne dass Absicherung durch Fremdbeurteilungen oder diagnostische Urteile zum Tragen gekommen sind. Dennoch erschien es legitim, bei der Herleitung der Fragestellung der Untersuchung als auch bei der Diskussion der Ergebnisse Studien zu Kindern und Jugendlichen mit ADHS (-Diagnose) einzubeziehen, da verschiedene Autoren (u. a. Jenni, 2017) darauf hinweisen, dass ADHS-Symptome kontinuierlich in der Population verteilt sind und ein Spektrum darstellen. Zudem zeigen Untersuchungen (z. B. Whalen et al., 2002), dass viele Jugendliche Einschränkungen aufweisen, auch wenn ihre ADHS-Symptome im subklinischen Bereich liegen.

In die Regressionsanalyse II wurde das generelle Vorhandensein von emotionaler Unterstützung durch Bezugspersonen sowie die wahrgenommene emotionale Unterstützung durch Mitschülerinnen und Mitschüler und Lehrpersonen einbezogen. Hierbei kann nicht ausgeschlossen werden, dass gewisse Überschneidungen vorliegen und für die Jugendlichen z. B. auch bestimmte Mitschülerinnen oder Mitschüler wichtige Bezugspersonen sind. Weiter wurden aus statistischen Gründen zwei separate Regressionsanalysen durchgeführt. Jedoch sind die beiden darin untersuchten Schutzfaktoren (Kompetenzerleben und emotionale Unterstützung) nicht unabhängig voneinander bzw. korrelieren zwischen $r=.11$ und .16. Die Ergebnisse eines Gesamtregressionsmodells mit allen Prädiktoren wären vermutlich leicht anders ausgefallen.

Bezüglich Ergebnissen muss festgehalten werden, dass die zusätzlich durch die Interaktion aufgeklärte Varianz in der multiplen Regressionsanalyse I klein ausfällt (nicht mehr als $1 \%$ der Gesamtvarianz). Dies wirft die Frage nach der praktischen Relevanz des Ergebnisses auf. Verschiedene Autoren (u. a. Judd et al., 2014; Luthar, 1993) weisen jedoch darauf hin, dass 
die Feststellung jeglicher Interaktionseffekte in nicht-experimenteller Forschung, insbesondere nach der Kontrolle für verschiedene Variablen (einschließlich der interagierenden) in früheren Schritten der Regressionsanalyse, bemerkenswert ist, da statistische Interaktionsterme aus verschiedenen methodischen Gründen sehr oft mit kleinen Effektgrößen verbunden sind. So zeigt sich beispielsweise auch in einer Überblicksarbeit von Dvorsky und Langberg (2016), dass nur sehr wenige Studien, die sich mit dem Thema Schutzfaktoren bei Kindern und Jugendlichen mit ADHS beschäftigten, signifikante Interaktionsterme aufdecken konnten. Ein Grund für die reduzierte Teststärke bei Interaktionseffekten liegt in der eingeschränkten Varianz innerhalb von Prädiktorvariablen. Ein Großteil der Jugendlichen (83,7\% der jungen Frauen und 76,4\% der jungen Männer) in der schulbasierten Stichprobe gaben im Durchschnitt auf der ADHS-Skala „gar keine“ bis „ein wenig" Symptome an. Ev. hätte man bei einer anderen Stichprobenzusammensetzung (viele Personen an beiden Polen der Symptomstärke) einen stärkeren Interaktionseffekt gefunden.

Die vorliegenden Querschnittdaten lassen keine Rückschlüsse auf kausale Wirkungen zu und die Resultate bedürfen der Absicherung durch längsschnittliche Untersuchungen. Obwohl die Wirkrichtung nicht untersucht werden konnte, deuten die Ergebnisse im Kontext anderer Studien, die einen Einfluss des Kompetenzerlebens und der wahrgenommenen sozialen Unterstützung auf das emotionale Wohlbefinden im Laufe der Zeit zeigen (Demaray et al., 2005; Rueger, Malecki \& Demaray, 2010), darauf hin, dass die signifikanten Faktoren im Regressionsmodell der vorliegenden Untersuchung als kompensatorisch bzw. protektiv aufgefasst werden können. Insgesamt wären für die Resilienzforschung bei Jugendlichen mit ADHSSymptomen theoriegeleitete und groß angelegte prospektive Längsschnittstudien von Nutzen, die spezifische Entwicklungsschritte von betroffenen Jugendlichen sowie relevante Schutzfaktoren aus einer entwicklungspsychopathologischen Perspektive untersuchen würden.

\section{Danksagung}

Die Autorin dankt Claudia Schellenberg, Pia GeorgiTscherry und Matthias Pfiffner für ihre Arbeit im Projekt und Katja Mackowiak für die hervorragende Betreuung.

\section{Anmerkungen}

1 Als Maturität wird in der Schweiz die Hochschulzugangsberechtigung bezeichnet.

2 Das eidgenössische Fähigkeitszeugnis EFZ ist eine anspruchsvolle drei- bis vierjährige Berufslehre. Parallel dazu kann eine Berufsmaturität erlangt werden.

3 Das eidgenössische Berufsattest (EBA) ist eine zweijährige Berufslehre, die zu einem eidgenössisch anerkannten Abschluss führt. Sie dient der Vermittlung von Qualifikationen zur Ausübung eines Berufs mit einfacheren Anforderungen.

4 Da der Nachweis von Interaktionseffekten in nichtexperimenteller Forschung u. a. wegen der schwachen statistischen Power sehr schwer ist (Judd, Yzerbyt \& Muller, 2014; McClelland \& Judd, 1993), wurden zwei separate Modelle gerechnet, um die Anzahl der in den Modellen enthaltenen Prädiktoren zu begrenzen.

Ein Schutzfaktor wirkt kompensatorisch, wenn er direkt mit einer geringeren Wahrscheinlichkeit eines negativen Outcomes einhergeht. Die günstige Wirkung auf das Individuum ist hierbei unabhängig vom Vorhandensein eines Risikofaktors. Beispielsweise geht soziale Unterstützung grundsätzlich bei allen Jugendlichen mit erhöhtem Wohlbefinden einher (Chu, Saucier \& Hafner, 2010). Von einem protektiven Schutzfaktor wird hingegen gesprochen, wenn ein Faktor die Wirkung eines Risikofaktors abpuffert. Der Schutzfaktor zeigt seine Wirkung nur bei Vorliegen eines Risikofaktors und moderiert somit die Beziehung zwischen Risikofaktor und Outcome. Typischerweise wird ein kompensatorischer Faktor mit einem direkten Haupteffekt eines Prädiktors und ein protektiver Faktor mit einem Interaktionseffekt in einer multiplen Regressionsanalyse nachgewiesen.

\section{Literatur}

Bachmann, C. J., Philipsen, A. \& Hoffmann, F. (2017) ADHD in Germany: Trends in Diagnosis and Pharmacotherapy. Deutsches Arzteblatt International, 114 (9), 141-148. https://doi.org/10.3238/arztebl.2017.0141

Bandura, A., Freeman, W. \& Lightsey, R. (1999). Self-efficacy: The exercise of control. Journal of Cognitive Psychotherapy, 13(2), 158-166. https://doi.org/10.1891/ 0889-8391.13.2.158

Baumgarten, F., Cohrdes, C., Schienkiewitz, A., Thamm, R. Meyrose, A. K. \& Ravens-Sieberer, U. (2019). Gesundheitsbezogene Lebensqualität und Zusammenhänge mit chronischen Erkrankungen und psychischen Auffälligkeiten bei Kindern und Jugendlichen. Ergebnisse aus KiGGS Welle 2. Bundesgesundheitsblatt Gesundheitsforschung Gesundheitsschutz, 62(10), 1205-1214. https://doi.org/10.1007/s00103-019-03006-9 
Bitto, H., Mörstedt, B., Faschina, S. \& Stieglitz, R.-D. (2017). ADHS bei Erwachsenen. Zeitschrift für Psychiatrie, Psychologie und Psychotherapie, 65(2), 121 131. https://doi.org/10.1024/1661-4747/a000311

Brooks, R. (2011). Building Resilience by Shaping Mindsets. In S. Goldstein, J.A. Naglieri \& M. DeVries (Eds.) Learning and Attention Disorders in Adolescence and Adulthood: Assessment and Treatment (pp. 367-404). Hoboken, New Jersey: John Wiley \& Sons.

Bullinger, M., Brutt, A. L., Erhart, M., Ravens-Sieberer, U. \& Bella Study Group. (2008). Psychometric properties of the KINDL-R questionnaire: results of the BELLA study. European Child \& Adolescent Psychiatry, 17 Suppl 1 (1), 125-132. https://doi.org/10.1007/s00787-008 1014-z

Casale, G., Hennemann, T. \& Hövel, D. (2014). Systematischer Überblick über deutschsprachige schulbasierte Maßnahmen zur Prävention von Verhaltensstörungen in der Sekundarstufe I. Empirische Sonderpädagogik, 6 33-58. https://doi.org/10.25656/01:9244

Castello, A. (2017). Schulische Inklusion bei psychischen Auffälligkeiten. Stuttgart: Kohlhammer Verlag.

Chu, P. S., Saucier, D.A. \& Hafner, E. (2010). Meta-Analysis of the Relationships Between Social Support and Well-Being in Children and Adolescents. Journal of Social and Clinical Psychology, 29(6), 624-645. https:// doi.org/10.1521/jscp.2010.29.6.624

Cohen, J., Cohen, P., West, S. G. \& Aiken, L. S. (2014). Applied Multiple Regression/Correlation Analysis for the Behavioral Sciences (2nd edition). New York: Psychology Press.

Cohen, S. \& Wills, T. A. (1985). Stress, social support, and the buffering hypothesis. Psychological Bulletin, 98, 310. https://doi.org/10.1037/0033-2909.98.2.310

Cole, D. A., Jacquez, F. M. \& Maschman, T. L. (2001). Social origins of depressive cognitions: A longitudinal study of self-perceived competence in children. Cognitive Therapy and Research, 25 (4), 377-395. https:// doi.org/10.1023/A:1005582419077

Currie, C., Inchley, J., Molcho, M., Lenzi, M., Veselska, Z. \& Wild, F. (2014). Health Behaviour in School-aged Children (HBSC) Study Protocol: Background, Me thodology and Mandatory items for the 2013/14 Survey. St. Andrews: Child and Adolescent Health Research Unit (CAHRU).

Dawson, J. F. (2014). Moderation in management research: What, why, when, and how. Journal of Business and Psychology, 29(1), 1-19. https://doi.org/10.1007/s108 69-013-9308-7

Demaray, M. K., Malecki, C. K., Davidson, L. M., Hodgson, K. K. \& Rebus, P. J. (2005). The relationship between social support and student adjustment: A longitudinal analysis. Psychology in the Schools, 42, 691-706. https://doi.org/10.1002/pits.20120

Döpfner, M. \& Görtz-Dorten, A. (2017). Diagnostik-System für psychische Störungen nach ICD-10 und DSM-5 für Kinder-und Jugendliche. Bern: Hogrefe.

Dvorsky, M. R. \& Langberg, J. M. (2016). A Review of Factors that Promote Resilience in Youth with ADHD and ADHD Symptoms. Clinical Child and Family Psychology Review, 19(4), 368-391. https://doi.org/10.1007/ s10567-016-0216-z

Dvorsky, M. R., Langberg, J. M., Becker, S. P. \& Evans, S. W. (2019). Trajectories of global self-worth in adolescents with ADHD: Associations with academic, emotional, and social outcomes. Journal of Clinical Child \& Adole scent Psychology, 48(5), 765-780. https://doi.org/10. 1080/15374416.2018.1443460
Edwards, G., Barkley, R. A., Laneri, M., Fletcher, K. \& Metevia, L. (2001). Parent-adolescent conflict in teenagers with ADHD and ODD. Journal of Abnormal Child Psychology, 29, 557-572. https://doi.org/10.1023/A:10 12285326937

Fabiano, G.A. \& Pyle, K. (2019). Best practices in school mental health for attention-deficit/hyperactivity disorder: A framework for intervention. School Mental Health, 11 (1), 72-91. https://doi.org/10.1007/s123 10-018-9267-2

Falkai, P. \& Wittchen, H. U. (2015). Diagnostisches und Statistisches Manual (DSM-5). Göttingen: Hogrefe.

Fergus, S. \& Zimmerman, M. A. (2005). Adolescent resilience: a framework for understanding healthy development in the face of risk. Annual Review of Public Health, 26, 399-419. https://doi.org/10.1146/annurev.publ health.26.021304.144357

Field, A. (2018). Discovering Statistics Using IBM SPSS Statistics (5th ed.). London: Sage Publications.

Frazier, T. W., Youngstrom, E. A., Glutting, J. J. \& Watkins, M. W. (2007). ADHD and achievement: meta-analysis of the child, adolescent, and adult literatures and a concomitant study with college students. Journal of Learning Disabilities, 40, 49-65. https://doi.org/10.1177 /00222194070400010401

Haft, S. L., Chen, T., LeBlanc, C., Tencza, F. \& Hoeft, F. (2019). Impact of mentoring on socio-emotional and mental health outcomes of youth with learning disabilities and attention-deficit hyperactivity disorder. Child and Adolescent Mental Health, 24 (4), 318-328. https://doi.org/10.1111/camh.12331

Hamre, B. K. \& Pianta, R. C. (2001). Early teacher-child relationships and the trajectory of children's school outcomes through eighth grade. Child Development, 72(2), 625-638. https://doi.org/10.1111/1467-86 24.00301

Hanisch, C., Casale, G., Volpe, R. J., Briesch, A. M. Richard, S., Meyer, H. et al. (2019). Gestufte Förderung in der Grundschule. Prävention und Gesundheitsförderung, 14(3), 237-241. https://doi.org/10.1007/ s11553-018-0700-z

Hurrelmann, K. (2007). Eine Einführung in die sozialwissenschaftliche Jugendforschung (11., vollst. überarb. Aufl.) Weinheim: Beltz Juventa.

Jenni, O. (2017). ADHS Spektrum. Lernen und Lernstörungen, 6, 113-121. https://doi.org/10.1024/2235-09 $77 / \mathrm{a} 000174$

Jia, M., Jiang, Y. \& Mikami, A. Y. (2016). Positively biased self-perceptions in children with ADHD: Unique predictor of future maladjustment. Journal of Abnormal Child Psychology, 44, 575-586. https://doi.org/10.10 07/s10802-015-0056-1

Judd, C. M., Yzerbyt, V.Y. \& Muller, D. (2014). Mediation and Moderation. In H.T. Reis \& C. M. Judd (Eds.) Handbook of Research Methods in social and Personality Psychology (Vol. 2, pp. 653-676). Cambridge: Cambridge University Press.

Keyes, C.L. (2013). Promoting and protecting positive mental health: Early and often throughout the lifespan. In C. L. Keyes (Eds.), Mental Well-being (pp. 3-28). Dordrecht: Springer.

Kienle, R., Knoll, N. \& Renneberg, B. (2006). Soziale Ressourcen und Gesundheit: soziale Unterstützung und dyadisches Bewältigen. In B. Renneberg \& P. Hammelstein (Hrsg.), Gesundheitspsychologie (S. 107-122). Heidelberg: Springer 
Kohn, J. \& Esser, G. (2008). ADHS im Jugend- und Erwachsenenalter. Monatsschrift Kinderheilkunde, 156(8), 748-756. https://doi.org/10.1007/s00112-008-17 31-x

Krueger, M. \& Kendall, J. (2001). Descriptions of self: an exploratory study of adolescents with ADHD. Journal of Child and Adolescent Psychiatric Nursing, 14(2), 61-72. https://doi.org/10.1111/j.1744-6171.2001. tb00294.x

Lampert, T., Hoebel, J., Kuntz, B., Müters, S. \& Kroll, L. E. (2018). Messung des sozioökonomischen Status und des subjektiven sozialen Status in KiGGS Welle 2. Journal of Health Monitoring, 3(1), 114-133. https:// doi.org/10.17886/RKI-GBE-2018-016

Laucht, M. (2012). Resilienz im Entwicklungsverlauf von der frühen Kindheit bis zum Erwachsenenalter - Ergebnisse der Mannheimer Risikokinderstudie. Frühförderung Interdisziplinär (3), 111-119. https://doi.org/10. 2378/fi_2012.art08d

Lee, S. S., Lahey, B. B., Owens, E. B. \& Hinshaw, S. P. (2008). Few preschool boys and girls with ADHD are well-adjusted during adolescence. Journal of Abnormal Child Psychology, 36, 373-383. https://doi.org/10.1007/s10 802-007-9184-6

Linderkamp, F., Hennig, T. \& Schramm, S. A. (2011). ADHS bei Jugendlichen: Das Lerntraining LeJA. Weinheim: Beltz.

Luthar, S. S. (1993). Methodological and conceptual issues in research on childhood resilience. Journal of Child psychology and Psychiatry, 34, 441-453.

Major, A., Martinussen, R. \& Wiener, J. (2013). Self-efficacy for self-regulated learning in adolescents with and without attention deficit hyperactivity disorder (ADHD) Learning and Individual Differences, 27, 149-156. https://doi.org/10.1016/j.lindif.2013.06.009

Martel, M., Nikolas, M. \& Nigg, J.T. (2007). Executive function in adolescents with ADHD. Journal of the American Academy of Child and Adolescent Psychiatry, 46(11), 1437 1444. https://doi.org/10.1097/chi.0b013e31814cf953

Masten, A. S. (2001). Ordinary magic. Resilience processes in development. American Psychologist, 56, 227-238. https://doi.org/10.1037//0003-066x.56.3.227

Mastoras, S. M., Saklofske, D. H., Schwean, V. L. \& Climie, E. A. (2018). Social support in children with ADHD An exploration of resilience. Journal of Attention Disorders, 22(8), 712-723. https://doi.org/10.1177/1087 054715611491

McClelland, G. H. \& Judd, C. M. (1993). Statistical difficulties of detecting interactions and moderator effects. Psychological Bulletin, 114, 376-390. https://doi.org/ 10.1037/0033-2909.114.2.376

McQuade, J. D., Hoza, B., Waschbusch, D. A., MurrayClose, D. \& Owens, J.S. (2011). Changes in self-perceptions in children with ADHD: a longitudinal study of depressive symptoms and attributional style. Beha vior Therapy, 42(2), 170-182. https://doi.org/10 1016/j.beth.2010.05.003

McQuade, J. D., Vaughn, A. J., Hoza, B., Murray-Close, D., Molina, B. S., Arnold, L. E. et al. (2014). Perceived social acceptance and peer status differentially predict adjustment in youth with and without ADHD. Journal of Attention Disorders, 18, 31-43. https://doi.org/10. $1177 / 1087054712437582$

Meinzer, M. C., Lewinsohn, P. M., Pettit, J. W., Seeley, J. R., Gau, J. M., Chronis-Tuscano, A. et al. (2013). Attention-deficit/hyperactivity disorder in adolescence pre- dicts onset of major depressive disorder through early adulthood. Depression and Anxiety, 30 (6), 546-553. https://doi.org/10.1002/da.22082

Molina, B. S., Hinshaw, S. P., Swanson, J. M., Arnold, L. E., Vitiello, B., Jensen, P. S. et al. (2009). The MTA at 8 years: prospective follow-up of children treated for combined-type ADHD in a multisite study. Journal of the American Academy of Child \& Adolescent Psychiatry, 48(5), 484-500. https://doi.org/10.1097/CHI.0b0 $13 \mathrm{e} 31819 \mathrm{c} 23 \mathrm{~d} 0$

Murray-Close, D., Hoza, B., Hinshaw, S. P., Arnold, L. E., Swanson, J., Jensen, P. S. et al. (2010). Developmental processes in peer problems of children with attentiondeficit/hyperactivity disorder in the Multimodal Treatment Study of Children With ADHD: developmental cascades and vicious cycles. Development and Psychopathology, 22, 785-802. https://doi.org/10.1017/S09 54579410000465

Noeker, M. \& Petermann, F. (2008). Resilienz: Funktionale Adaptation an widrige Umgebungsbedingungen. Zeitschrift für Psychiatrie, Psychologie und Psychotherapie, 56 (4), 255-263. https://doi.org/10.1024/1661-4747.56. 4.255

Ostrander, R. \& Herman, K. C. (2006). Potential cognitive, parenting, and developmental mediators of the relationship between ADHD and depression. Journal of Consulting and Clinical Psychology, 74, 89-98. https://doi. org/10.1037/0022-006X.74.1.89

Philipp-Wiegmann, F., Rösler, M., Retz, W., Schindelbeck, K. \& Retz-Junginger, P. (2015). Häufigkeiten von adulten ADHS-assoziierten Symptomen in der Normalbevölkerung - Referenzwerte der ADHS-Selbstbeurteilungsskala und des Wender-Reimherr-Interviews. Zeitschrift für Psychiatrie, Psychologie und Psychotherapie, 63(4), 267-278. https://doi.org/10.1024/1661-4747/ a000249

Powell, V., Riglin, L., Hammerton, G., Eyre, O., Martin, J., Anney, R. et al. (2020). What explains the link between childhood ADHD and adolescent depression? Investigating the role of peer relationships and academic attainment. European Child \& Adolescent Psychiatry, 29, 1581-1591. https://doi.org/10.1007/s00787-019-01 463-w

Rademacher, C., Walter, D. \& Döpfner, M. (2002). SELBST - Ein Therapieprogramm zur Behandlung von Jugendlichen mit Selbstwert, Aktivitäts- und Affekt-, Leistungsund Beziehungsstörungen. Kindheit und Entwicklung, 11, 107-118. https://doi.org/10.1026//0942-5403. 11.2.107

Ravens-Sieberer, U. \& Bullinger, M. (2003). Der Kindl-RFragebogen zur Erfassung der gesundheitsbezogenen Lebensqualität bei Kindern und Jugendlichen - Revidierte Form. In J. Schumacher, A. Klaiberg \& E. Brähler (Hrsg.), Diagnostische Verfahren zu Lebensqualität und Woblbefinden (S. 184-188). Göttingen: Hogrefe.

Ravens-Sieberer, U., Wille, N., Bettge, S. \& Erhart, M. (2007). Psychische Gesundheit von Kindern und Jugendlichen in Deutschland. Ergebnisse aus der BELLAStudie im Kinder- und Jugendgesundheitssurvey (KiGGS) Bundesgesundheitsblatt Gesundheitsforschung Gesundheitsschutz, 50(5-6), 871-878. https://doi. org/10.1007/s00103-007-0250-6

Ringdal, R., Espnes, G.A., Eilertsen, M.-E. B., Bjørnsen, H. N. \& Moksnes, U. K. (2020). Social support, bullying, school-related stress and mental health in adolescence. Nordic Psychology, 72(4), 313-330. https:// doi.org/10.1080/19012276.2019.1710240 
Ritter, M., Bilz, L. \& Melzer, W. (2016). Schulische und außerschulische Unterstützung als Ressource für die psychische Gesundheit von Schülerinnen und Schülern. In L. Bilz, G. Sudeck, J. Bucksch, A. Klocke, P. Kolip, W. Melzer, U. Ravens-Sieberer \& M. Richter (Hrsg.), Schule und Gesundheit: Ergebnisse des WHOJugendgesundheitssurveys "Health behaviour in schoolaged children "(S. 181-199). Weinheim: Beltz Juventa.

Rotter, J. B. (1966). Generalized expectancies for interna versus external control of reinforcement. Psychological Monographs, 80(1), 1-28. https://doi.org/10.1037/h0 092976

Rueger, S. Y., Malecki, C. K. \& Demaray, M. K. (2010). Relationship between multiple sources of perceived social support and psychological and academic adjustment in early adolescence: Comparisons across gender. Journal of youth and adolescence, 39, 47. https://doi. org/10.1007/s10964-008-9368-6

Rueger, S. Y., Malecki, C. K., Pyun, Y., Aycock, C. \& Coyle, S. (2016). A meta-analytic review of the association between perceived social support and depression in childhood and adolescence. Psychological Bulletin, 142, 1017-1067. https://doi.org/10.1037/bul0000058

Sarubin, N., Gutt, D., Giegling, I., Bühner, M., Hilbert, S., Krähenmann, O. et al. (2015). Erste Analyse der psychometrischen Eigenschaften und Struktur der deutschsprachigen 10- und 25-Item-Version der Connor-Davidson Resilience Scale (CD-RISC). Zeitschrift für Gesundheitspsychologie, 23, 112-122. https://doi. org/10.1026/0943-8149/a000142

Schei, J., Nøvik, T.S., Thomsen, P. H., Indredavik, M.S. \& Jozefiak, T. (2015 a). Improved quality of life among adolescents with attention-deficit/hyperactivity dis order is mediated by protective factors: a cross sectional survey. BMC psychiatry, 15(1), 108. https://doi.org/ 10.1186/s12888-015-0491-0

Schei, J., Nøvik, T. S., Thomsen, P. H., Lydersen, S., Indredavik, M.S. \& Jozefiak, T. (2015 b). What Predicts a Good Adolescent to Adult Transition in ADHD? The Role of Self-Reported Resilience. Journal of attention disorders, 22, 547-560. https://doi.org/10.1177/1087 054715604362

Schellenberg, C., Krauss, A., Pfiffner, M. \& Georgi-Tscherry, P. (2020). Inklusive Didaktik und Nachteilsausgleich an Berufsfachschulen und Gymnasien. Ergebnisse de Forschungsprojektes „Enhanced Inclusive Learning“. Schweizer Zeitschrift für Heilpädagogik, 26(7-8), 17-26.

Schmidt, S., Brähler, E., Petermann, F. \& Koglin, U. (2012). Komorbide Belastungen bei Jugendlichen und jungen Erwachsenen mit ADHS. Zeitschrift für Psy- chiatrie, Psychologie und Psychotherapie, 60(1), 15-26. https://doi.org/10.1024/1661-4747/a000094

Schulz, U. \& Schwarzer, R. (2003). Soziale Unterstützung bei der Krankheitsbewältigung: Die Berliner Social Support Skalen (BSSS). Diagnostica, 49(2), 73-82. https://doi.org/10.1026//0012-1924.49.2.73

Schwab, S. \& Fingerle, M. (2013). Resilienz, Ressourcenorientierung und Inklusion. In S. Schwab, M. Gebhardt, E. Ederer-Fick \& B. Gasteiger-Klicpera (Hrsg.), Theorien, Konzepte und Anwendungsfelder der inklusiven Pädagogik (S. 97-108). Wien: Facultas.wuv.

Schwarzer, R. \& Luszczynska, A. (2006). Self-efficacy, adolescents' risk-taking behaviors, and health. In F. Pajares \& T. Urdan (Hrsg.), Self-efficacy beliefs of adolescents (Bd. 5, S. 139-159). Greenwich, Connecticut: Information Age Publishing.

Tischler, L., Schmidt, S., Petermann, F. \& Koglin, U. (2010). ADHS im Jugendalter: Symptomwandel und Konsequenzen für Forschung und klinische Praxis. Zeitschrift für Psychiatrie, Psychologie und Psychotherapie, 58 (1), 23-34. https://doi.org/10.1024/1661-4747.a000003

Torsheim, T., Wold, B. \& Samdal, O. (2016). The Teache and Classmate Support Scale. School Psychology International, 21, 195-212.https://doi.org/10.1177/01430 34300212006

Vieno, A., Santinello, M., Pastore, M. \& Perkins, D. D. (2007). Social support, sense of community in school, and self-efficacy as resources during early adolescence: an integrative model. American Journal of Community Psychology, 39, 177-190. https://doi.org/10.1007/s10 464-007-9095-2

Werner, E. E. (2004). Journeys from childhood to midlife: risk, resilience, and recovery. Pediatrics, 114, 492. https://doi.org/10.1542/peds.114.2.492a

Whalen, C. K., Jamner, L. D., Henker, B., Delfino, R. J. \& Lozano, J. M. (2002). The ADHD spectrum and everyday life: Experience sampling of adolescent moods, activities, smoking, and drinking. Child Development, 73, 209-227. https://doi.org/10.1111/1467-8624.00401

\section{Annette Krauss}

Institut für Verhalten, sozio-emotionale und psychomotorische Entwicklungsförderung (IVE) Interkantonale Hochschule für Heilpädagogik Schaffhauserstr. 239

Postfach 5850

$\mathrm{CH}-8050$ Zürich

E-Mail: annette.krauss@hfh.ch 